\title{
22 Relationships between the spatial and theoretical in geometry: the role of computer dynamic representations in problem solving
}

\author{
Colette Laborde \\ Laboratoire Leibniz-IMAG \\ Grenoble, France
}

\begin{abstract}
Diagrams play an important role in geometry teaching. An analysis of tasks and students' behaviour in solving problems in two different environments, paper-andpencil or computer, indicated that the dynamic nature of the software changes the relationship between diagrams and the theoretical aspects of the subject. Learning geometry seems to involve not only learning how to use theoretical statements in deductive reasoning, but also learning to recognise visually relevant spatialgraphical invariants attached to geometrical invariants. Observations of students revealed that this was not easy for them-moving between the spatial and the theoretical domains was not spontaneous and they tended to consider each domain independently.
\end{abstract}

\section{Keywords}

Geometry, software, direct manipulation, problem solving, case studies.

\section{THE DISTINCTION BETWEEN SPATIAL AND THEORETICAL PROPERTIES}

Physical space and geometry as a set of theories are two separate domains: space is considered here as part of reality while geometry partly models space and also develops its own questions and answers.

Diagrams in two-dimensional geometry play an ambiguous role: on the one hand, they refer to theoretical objects while on the other, they offer graphicalspatial properties which can give rise to a student's perceptual activity. This

Information and Communications Technologies in School Mathematics

J.D. Tinsley \& D.C. Johnson (Eds.)

(c) 1998 IFIP. Published by Chapman \& Hall 
ambiguous role of diagrams is completely implicit in traditional geometry teaching where it is assumed that it is possible simply to abstract the properties of the theoretical object represented by the diagram so theoretical properties are assimilated into graphical ones. One of the consequences of this assumption is that students often conclude that it is possible to construct a geometrical diagram using only visual cues, or to deduce a property empirically by checking the diagram. When students are asked by a teacher to construct a diagram, the teacher expects them to work at the level of geometry using theoretical knowledge, whereas students very often stay at a graphical level and try only to satisfy the visual constraints.

In these circumstances, rather than helping students, diagrams become an obstacle to geometrical thinking in the sense that they allow students to avoid reasoning in theoretical terms (Fishbein, 1990, Mariotti, 1995, Salin and Berthelot, 1994, Duval, 1995). This leads to the question of the difference between what we call spatial-graphical and theoretical properties in geometry.

Some spatial-graphical properties of the diagram are incidental to the geometrical problem while others are necessary. Further, spatial-graphical properties necessarily follow from others: e.g., there is a necessary link between the parallelism of sides of a quadrilateral and the fact that its diagonals intersect in their midpoint. In theory, geometry does not consider the slope of the sides of the quadrilateral as relevant but does consider as relevant the identity of the slopes of those sides.

The teaching of geometry deals with these necessary links between spatialgraphical properties, but one can understand the nature of these links if and only if one also can understand that some other links are merely incidental. Necessity makes sense in opposition to contingence. Geometry may appear useful if it allows one to predict, to produce or to explain spatial-graphical properties of diagrams because of these necessary links, but it first requires an awareness of the distinction between such properties and those that are theoretical.

\section{MOVES BETWEEN THE THEORETICAL AND THE SPATIAL GRAPHICAL DOMAINS}

We start from the distinction between the domain of geometrical objects and relations (denoted by $T$, referring to theoretical) and that of spatial-graphical entities (denoted by SG) exemplified by diagrams on paper, on a computer screen or by movement produced by a linkage point of a machine. This distinction is very close to that of conceptual/figural, made by Fishbein (1993) and Mariotti $(1995,100-104)$. T denotes the theoretical referents in a geometrical theory, it refers to theoretical objects, relations and operations on these objects as well as to judgements about them that can be expressed in various languages; SG denotes the graphical entities on which it is possible to perform physical actions, and about which it is possible to express ideas, interpretations, opinions, judgements. 
School problems require the use of both domains and several moves between them. We think that this interplay between $T$ and SG is an essential part of the meaning of geometry (a claim also made by Mariotti (op. cit.)). We also consider that this back and forth process between T and SG takes place even for experts in school geometry, e.g. teachers. Figure 1 illustrates the activity of the problem solver according to this view in the case of a problem, which starts and ends in the $\mathrm{T}$ domain. After recognising a known configuration on the diagram the solver interprets it in theoretical terms and may deduce a geometrical property by means of theoretical considerations (theorem or definition). The solver may come back to the diagram with this gained information and go further in the analysis of the diagram ...

Theoretical (T)

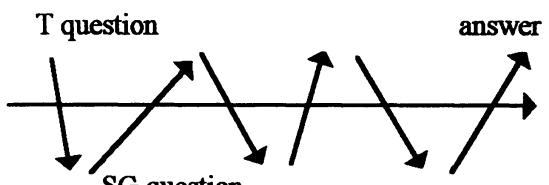

Spatial-graphical (SG)

SG question

result

Figure 1

\section{DIAGRAMS IN COMPUTER BASED ENVIRONMENTS}

Spatial-graphical and geometrical aspects are very much interrelated in the new kind of diagrams provided by geometry microworlds because their behaviour is controlled by theory. In dynamic geometry microworlds, such as Cabri-géomètre (Laborde and Straesser, 1990) or Geometer Sketchpad (Jackiw, 1993), diagrams result from sequences of primitives expressed in geometrical terms chosen by the user. When an element of such a diagram is dragged by the mouse, the diagram is modified while all the geometric relations used in its construction are preserved. These artificial realities can be compared to entities of the real world. It is as if they react to the manipulations of the user by following the laws of geometry, just like material objects react by following the laws of physics. A crucial feature of these realities is their quasi-independence of the user once they have been created. When the user drags one element of the diagram, it is modified according to the geometry of its constructions rather than the wishes of the user. This is not the case in paper and pencil diagrams which can be slightly distorted by the students in order to meet their expectations.

Computers diagrams are also external objects whose behaviour and feedback requires interpretation by the students. Geometry is one means, among others, of interpreting this behaviour (Balacheff and Sutherland, 1994). Visualisation plays a critical role in this process. As Dreyfus (1993) claims, computer-based environments promote visually exploratory behaviours and as such may have a considerable influence on both research in mathematics and in mathematics education. It can be easily assumed that these geometry microworlds favour a 
stronger link between spatial-graphical and geometrical aspects since spatial invariants in the moving diagrams almost certainly represent geometrical invariants. We will attempt to identify in the following section the extent to which this closer link might affect students' solutions.

\section{INTERRELATIONS BETWEEN DIAGRAMS AND GEOMETRY IN STUDENTS' SOLVING PROCESSES}

Our aim was to study the moves between T and SG empirically, by giving students geometry tasks and analysing their verbalisations and actions. This approach is close to that of Bartolini Bussi (1991, II-100) who distinguished two types of moves in the solution process of students involved in a geometrical task based on linkages: experimental moves and logical moves. An experimental move is based on visual tactile experiment while a logical move involves the production of a statement deduced from accepted statements. Because we aim to focus on the links between diagrams and theory, our method of coding the activity of the students considers, in addition, the moves between what Bussi would call experimental level and logical level.

\section{Method of investigation}

To investigate these interrelations, we observed students working in pairs on a joint task in geometry with Cabri-géomètre and in a paper and pencil environment. Their verbal exchanges allowed the researcher to work on the externalising of their approaches and ideas. Tasks from various categories (e.g. construction tasks, proof tasks) were used. Students were audio-taped and their verbal exchanges transcribed; all their actions on the computer (dragging an element of the diagram, use of a menu item, typing on the keyboard, click of the mouse) were also recorded by the facility called 'journal of session'.

Once a protocol comprising the transcription of the students' verbalisations and actions and the screen diagrams had been made, it was segmented into units each of which were ascribed to one of the following three categories: referring to spatial-graphical realities, SG; referring to geometrical objects and relation, T; establishing a link between T and SG.

All the work on protocols produced evidence of several phenomena which could well have remained invisible without it. Some of these phenomena, relating to the paper and pencil and computer environments, were:

- the meaning of the task for each student; without this systematic analysis, we would not have discovered that the task intended to deal with geometry was conceived as a spatial-graphical task in some work phases or even in the work throughout the whole task.

- some contract effects affecting the meaning of the task which may differ from one environment to another one; and 
- the importance of the influence of the software environment not only on the solving processes but also on the nature of the task itself.

\section{TWO ILLUSTRATIVE PROBLEMS}

The students' problem solving processes are illustrated here through the analysis of the protocols of their work on two problems, see figure 2 and figure 3 , in each of the two environments (pencil and paper or computer). Note that a pair of students worked on both problems in only one environment. In the case of pencil and paper, the students had a straight edge (ruler) and a set square and a compass available to them.

P1 Let $[\mathrm{AB}]$ be a segment, $d$ the perpendicular bisector of $[\mathrm{AB}], \mathrm{O}$ a point of the bisector. Draw the rectangle with centre $O$ and two vertices $A$ and $B$.

1. Explain how you constructed the rectangle (in paper and pencil environment).

2. Save your solution (in Cabri environment).

3. Justify your construction (in both environments).

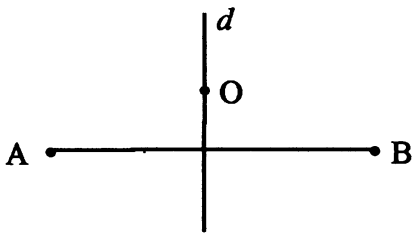

Figure 2 Problem 1.

P2 Let $d$ and $d^{\prime}$ be two intersecting straight lines and $\mathrm{P}$ a point which does not belong to any of those lines. Construct two points A and B such that A belongs to $d, \mathrm{~B}$ belongs to $d^{\prime}$ and $\mathrm{P}$ is the midpoint of [AB].

1. Explain how you constructed A and B (in paper and pencil environment).

2. Save your solution (in Cabri environment).

3. Justify your construction.

Figure 3 Problem 2.

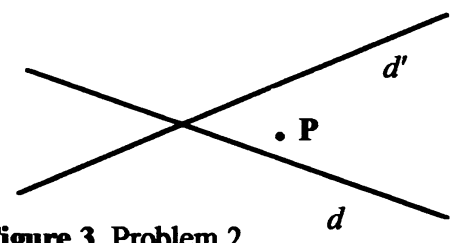

$d$

The two problems have been chosen to be investigated jointly because they are both construction problems and as such require working on a diagram using theoretical means. This kind of problem seemed to be especially adequate for an investigation on moves between diagram and theory. But both problems strongly differ in the availability of the spatial graphical solution. In problem 1, students predict very easily by eye the approximate spatial position of the rectangle. The only problem is to find the construction process based on a allowed use of instruments in a paper and pencil environment or on Cabri primitives in a Cabri 
environment. In problem 2, students are not able to predict the spatial position of $A$ and $B$ meeting the problem conditions. Problem 1 should be immediately a problem of $T$ domain for the students while problem 2 as it is stated could be a spatial problem as well as a theoretical problem.

The difference between the two problems can also be expressed in other terms by means of a metaphor of the duality between arithmetic and algebra: problem 1 could be qualified as of arithmetical nature while problem 2 as of algebraic nature. There are no variable objects involved in the system of relations to be found in problem 1: the rectangle may be directly determined from points A, B and $O$ just as the solution of an arithmetical problem evolves from the numbers given in the statement. In problem 2 , there are three variable objects, $d, d^{\prime}$ and $\mathrm{P}$, and two variable points $A$ and $B$, with the solutions to be determined using the following system of relationships:

\section{A belongs to $d$ \\ $P$ is the midpoint of $A$ and $B$ \\ B belongs to $d$}

One possible solution, based on a strategy of relaxing a constraint, is to vary $A$ on $d$ and to consider the locus of points $\mathrm{A}^{\prime}$ such that $\mathrm{P}$ is midpoint of $\mathrm{A}$ and $\mathrm{A}^{\prime}$. It is a straight line, with the image of $d$ through the point symmetry with centre $P$. Point $\mathrm{B}$ is the intersecting point of this line and of $d^{\prime}$. This solution process is similar to using substitution in finding the solution for an algebraic linear system with two unknowns.

Note that problem 2 may be turned into an arithmetical problem very close to problem 1 if it would have been stated as such: Let $d$ and $d^{\prime}$ be two straight lines intersecting into $O$ and a point $P$. Construct the parallelogram $A O B C$ with centre $P$ so that A belongs to $d$ and $\mathrm{B}$ to $d^{\prime}$. It is the kind of solution which may be found if a rough sketch of the solution is drawn and if the figure is analysed (the old well-known process of analysis and synthesis).

\section{Meaning of the task}

A construction task given by the teacher as a T problem is not necessarily viewed as such by the students as mentioned at the beginning of this paper. Students could see the solving process of a construction problem as consisting of two successive and separate phases: in the first phase they have to find the spatial answer, in a second phase because of the classroom context (didactical contract) they have to find a justification in theoretical terms for the spatial answer. Theory is not used as providing the means for solving, but rather is required in order to meet the expectations of the teacher. Actually this was reinforced in problems 1 and 2 by the sequence of questions 1 or 2 (construction process) and 3 (justification). Nevertheless in question 1 of problem 1 it was expected that students would perform actions of drawing by using the theoretical properties of the rectangle, which should be familiar to them. So we did not expect a separation of spatial level and theoretical level in this question. 
Actually we did not observe any real work at the $T$ level for this question. The most common construction strategy in both environments was to construct the sides of the rectangle-two perpendicular lines to the segment $[A B]$ at points $A$ and $\mathrm{B}$ and then the fourth side as perpendicular to line $d$ through a point equidistant from the line segment and the new perpendicular (see figure 4 overleaf).

During this drawing phase, very little knowledge about the rectangle was made explicit, they drew perpendiculars and the fourth side without actually referring to any particular properties of the rectangle. They expressed actions like "draw a perpendicular line", or "make a perpendicular line". The only mathematical words used were: perpendicular, parallel and equal distance. One can assume that these geometrical properties were completely embedded in their discourse about actions since the control they had on their production was purely of visual nature. Some students in their trials rejected constructions by saying: "it does not look a rectangle".

In the construction task, the students were attracted by the shape (the sides) of the rectangle and not by the vertices. So they very seldom used the diagonals, and only after obtaining the rectangle by its edge using perpendiculars did some of the students extend segments $\mathrm{AO}$ and $\mathrm{BO}$ by the same length in order to obtain the two other vertices of the rectangle, even though this was the more economic strategy in terms of number of operations to perform (especially in Cabri-géomètre).

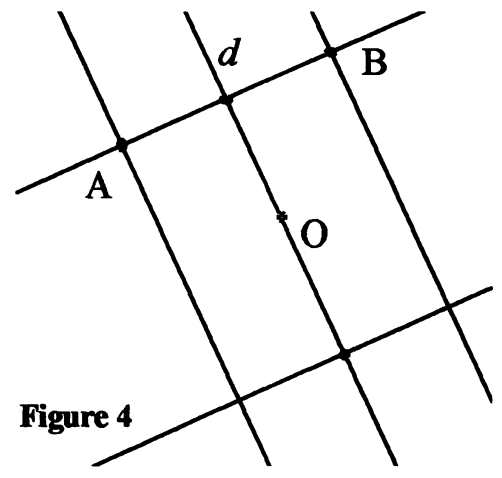
However, during the phase of justification, because the problem statement mentioned that $O$ is the centre of the rectangle to draw, almost all the students justified their construction by referring to the theorem of the intersecting point of the diagonals of a parallelogram. The justification at the $T$ level did not really account for the drawing process. They were seeking a proof to satisfy the request for a justification: justifying in the culture of a mathematics class means proving by using geometrical reasons. The theorem of the midpoint of the diagonals seemed to them appropriate, in particular because it is the theorem about quadrilaterals in the teaching at this level of schooling.

In problem 2, all students tried first to obtain a spatial solution. But their behaviours differed very much in both environments. In the paper and pencil environment, they obtained a spatial answer by trial and error or just by eye and were satisfied, while in Cabri each of their spatial solutions (often obtained by dragging) was disqualified when they checked whether the solution was preserved under the drag mode. So all students spent a long time without finding a solution. Note that the paper and pencil students produced a justification which only described their drawing process by eye. The observations of problem 2 in both 
environments showed very little work at the $T$ level and almost no moves between SG and T.

\section{Some effects linked to the didactical content}

The break between finding the spatial solution and the theoretical justification was clearly made explicit in the verbal exchanges of some pairs solving problem 1.

Marie is expressing to Hugues : "we must justify by theorems we know"

Hugues replying : "théorème de Pythagore, de Thalès"

or Florence : "we'll explain by means of logic"

Sofien and Pauline after having drawn the rectangle and starting to reflect about the way of justifying had also an interesting dialogue.

Pauline reading the statement : "Justify your construction"

Sofien : "One makes the three perpendiculars to make the right angles"

Pauline : "No it is not this. As in a triangle ... in a rectangle the diagonals intersect in their midpoint ..."

Finding theoretical reasons is clearly seen as distinct from the construction problem (as it appears when Pauline rejected the description of Sofien). In the action of the students as well as in their discourse, it had no intrinsic necessity but was supposed to meet expectations of the teacher. It must follow some rules, i.e., the recourse to taught theorems. The theoretical nature is also expressed by the students' use of sentences such as "comme nous savons que ..." (as we know that ...) revealing that theory is linked to knowledge while construction is on the side of action.

Another strong contract effect occurred for both problems in the work of some students in the paper and pencil environment who found that they must use a compass in a construction problem. Surely the teacher was expecting something else than the use of only a straight edge and a set square.

The software environment breaks this noble status of compass inherited from the Euclidean tradition, placing in a democratic way, all geometric primitives at the same rank. We did not observe this search for the use of a circle for students working in the Cabri environment. But nevertheless we still observed the gap between the construction process and the theoretical reasons given in the justification phase.

\section{Richness and complexity of the software}

As indicated previously, feedback available on Cabri was often used by students, leading them to reject numerous ideas or trials, whereas in the paper and pencil environment students were quickly satisfied by what they thought to be a solution. Cabri is more demanding at geometrical level than paper and pencil. But the dynamic possibilities of Cabri while widening the scope of exploration may also 
have introduced a new complexity in the problem due to the possible variations of the given elements of the problem.

Problem 2 is representative of the kind of problems mixing given and variable elements. Students tried to obtain a spatial solution for A and B in considering any point $\mathrm{A}$ on $d$, any point $\mathrm{B}$ on $d^{\prime}$ and trying to superimpose lines AP and BP. But when stating that $P$ was not the midpoint of $[A B]$, they also moved $P$ until obtaining it coinciding with the midpoint of $[\mathrm{AB}]$. After obtaining this fragile solution they could not go further: every element was independent from others in this drawing. It was not possible to establish a relation between elements. The behaviours of the students was similar to that of beginners in algebra. Being confronted to a word problem, they denote every element of the statement by a letter without expressing algebraic relations between them. Hugues felt this way about the variability of the points and said: "we must find a trick in order to move simultaneously the two points" but never succeeded. The possibility of dragging introduced indeed two elements of complexity:

- it required distinguishing between the possibility of varying $P$ which was given (as a way of checking the robustness of the construction of A and B) and the variation of $A$ on $d$ (which had to be used in order to find the locus of the other endpoint of $[\mathrm{AB}])$;

- it allowed A and B to vary independently and prevented students from considering a relation between $\mathrm{A}$ and $\mathrm{B}$.

Overcoming the complexity requires without any doubt the intervention of the teacher. The didactical problem is that the teacher must intervene without substantially changing the task for the students, i.e. the Topaze effect (Brousseau, 1992).

A small experiment should be mentioned here. After students spent one hour on problem 2 without finding a correct solution, two pairs, one working with paper and pencil, the other one with Cabri, were given the hint that they should use a geometrical transformation. Paper and pencil students did not change their solution, whereas Cabri students decided to use point symmetry which they could read in the menus. This was the starting point of a long but successful process. Instead of looking for a spatial solution by moving, A and B independently, they constructed $B$ as image of $A$ in a point symmetry around $P$. They asked for the locus of B when A was moving on $d$. They recognised a straight line parallel to $d$ and from that in a rather awkward process they found a spatial solution. Analysing the spatial solution in geometrical terms, they extracted a geometrical construction as shown in figure 5 (opposite).

At this point, they had no geometrical reason for this construction, they simply stated that it worked and was resistant to the drag mode. When searching for a justification, after some experiments on the drawing, they recognised the configuration of midpoints in a triangle and understood the geometrical rationale for their pragmatic construction. This rather long description reveals how tedious the way to geometry was for the students and that the interplay between SG and T 
levels took place only after they had found a spatial solution: it occurred first when they extracted a geometrical construction from their drawing and second when they were searching for a geometrical justification of their pragmatic construction.

Draw a line parallel to $d^{\prime}$ and passing trough $P$. It intersects $d$ in $\mathrm{S}$.

Construct $A$ as the symmetrical point of $O$ around $\mathrm{S}$. Construct $\mathrm{B}$ as the symmetrical point of $\mathbf{A}$.

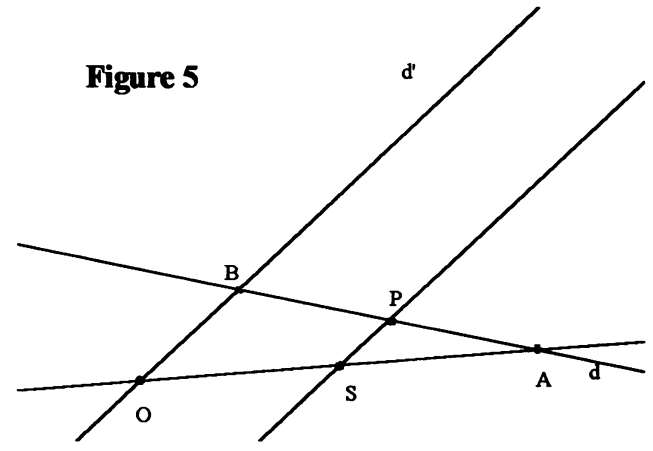

What also must be stressed, is the necessity for students to experience this first phase at the SG level before coming to the $T$ level. The geometrical problem makes sense for the students only after they could manipulate at the spatial level and perform several spatial experiments. From this point of view, the software environment differs substantially from the paper and pencil environment in which the spatial activity is inhibiting and clumsy (cost of drawing operation, difficulty of erasing, absence of dragging, etc.) and feedback is rather poor.

\section{CONCLUSION}

Analysing the tasks and the students' behaviours in terms of the existence of two domains of objects, the spatial-graphical domain and the geometrical/theoretical one, indicated that their coexistence was both a source of difficulties and ambiguities as well as a source with the potential for enriching students' experiences. Learning geometry seems to involve not only learning how to use theoretical statements in deductive reasoning but also learning to recognise visually relevant spatial-graphical invariants attached to geometrical invariants. Observation of students revealed that this was not easy for them: moving between the spatial and the theoretical domains was not spontaneous and they tended to consider each domain independently. Contrasting the work of students in the paper and pencil environment and in the Cabri environment leads to several claims:

- the weakness of feedback offered in the paper and pencil environment inhibits students' movement between the graphical and theoretical domains; 
- while the software environment is more demanding, it offers a greater complexity, and may be a source of difficulties for the students; it also provides for making the links between the two domains.

Our more general claim would be that Cabri does not hide the complexity of the situation as the paper and pencil environment does. If the students overcome this complexity, they probably learn more. However, they do not automatically overcome this complexity. The didactical problem is one of explicating the role of the teacher in helping students cope with this complexity through interventions which facilitate the movement between the spatial-graphical and theoretical domains.

\section{REFERENCES}

Balacheff, N. and Sutherland, R. (1994). Epistemological domain of validity of microworlds: the case of Logo and Cabri-géomètre In R. Lewis and P. Mendelsohn (eds) Lessons from Learning. IFIP Transactions, A 46, 137150. Amsterdam: North Holland and Elsevier Science B.V.

Bartolini Bussi, M. (1991). Geometrical proofs and mathematical machines: an exploratory study, Proceedings of the XVIIth Conference of the International Group for Psychology of Mathematics Education. Tsukuba, Japan: University of Tsukuba, 97-104.

Brousseau, G. (1992). Didactique: what it can do for the teacher. Recherches en didactique des mathématiques. Selected Papers. 7-40.

Dreyfus, T. (1993). Didactic design of computer based learning environments. In C. Keitel and K. Ruthven (eds.), Learning from Computers. Nato ASI Series, Heidelberg: Springer Verlag, 101-130.

Duval, R. (1995). Geometrical pictures: kinds of representation and specific processings. In R. Sutherland and J. Mason Exploiting Mental Imagery with Computers in Mathematics Education. NATO ASI Series, Berlin, Heidelberg: Springer Verlag, 142-157.

Fishbein, E. (1993). The theory of figural concepts, Educational Studies in Mathematics, 24(2), 139-62.

Jackiw N. (1993). Geometer Sketchpad (1993) The Visual Geometry Project (software). Swarthmore College and Berkeley: Key Curriculum Press.

Laborde, J-M. and Straesser, R (1990). Cabri-géomètre: a microworld of geometry for guided discovery learning, Zentralblatt fuer Didaktik der Mathematik 5, 171-77.

Mariotti, M. (1995). Images and Concepts in Geometrical Reasoning. In R. Sutherland and J. Mason (eds.) Exploiting Mental Imagery with Computers in Mathematics Education. NATO ASI Series, Berlin, Heidelberg: Springer Verlag, 97-116. 
Salin, M-H. and Berthelot, R. (1994). Phénomènes liés á l'insertion de situations adidactiques dans l'enseignement élémentaire de la géomètrie. In $\mathbf{M}$. Artigue, R. Gras, C. Laborde and P Tavignot (eds.), Vingt ans de didactique des mathématiques en France. Grenoble: Editions La Pensée Sauvage, 275-82.

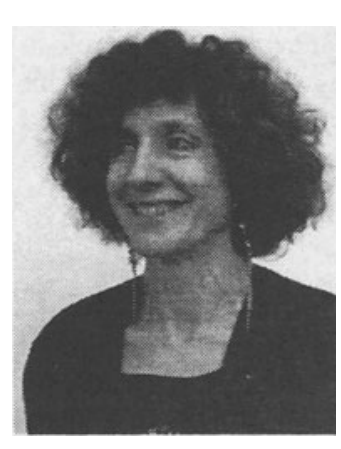

Colette Laborde graduated in mathematics from the Ecole Normale Supérieure. She obtained a 'doctorat ès sciences' in mathematics education at the Scientific University of Grenoble on the language problems in mathematics teaching and learning. She is a full professor at the university teacher institute and is member of the research group in computer environments and human learning at Leibniz laboratory (of the Institute of Applied Mathematics and Computer Sciences). She is involved in the project Cabri-géomètre. She is head of the doctoral program of mathematics and science education at the University of Grenoble. 